\title{
RESPONSE DUE TO IMPULSIVE FORCE IN GENERALIZED THERMO- MICROSTRETCH ELASTIC SOLID
}

\author{
V. KUMAR * \\ Department of Mathematics \\ Lovely Professional University, Punjab, INDIA \\ E-mail: varun.kumar@hotmail.co.in \\ R. SINGH \\ Department of Mathematics \\ S.G.A.D. Govt. College, Punjab, INDIA \\ E-mail:kalsi_ranjit@yahoo.com
}

\begin{abstract}
A two dimensional Cartesian model of a generalized thermo-microstretch elastic solid subjected to impulsive force has been studied. The eigen value approach is employed after applying the Laplace and Fourier transforms on the field equations for L-S and G-L model of the plain strain problem. The integral transforms have been inverted into physical domain numerically and components of normal displacement, normal force stress, couple stress and microstress have been illustrated graphically.
\end{abstract}

Key words: thermo-microstretch, eigen value, Laplace transform, Fourier transform, impulsive force.

\section{Introduction}

Inadequacy of the classical theory of elasticity to model the modern engineering components which possess internal structure such as polycrystalline materials and materials with fibrous or coarse grain structure led scientists to develop a new theory which can successfully explain the behavior of such materials. Eringen (1966) coined a term micropolar to explain deformation of elastic media with such oriented and interconnected particles made up of dipole atoms or dumb-bell molecules which are also capable of translational as well as rotational motion when subjected to surface and body couples.

The conventional coupled thermoelasticity theory based on a parabolic heat equation, which predicts an infinite speed for the propagation of heat leading to the conclusion that that if an isotropic, homogeneous, elastic material is subjected to thermal or mechanical disturbances; the effects in the temperature and displacement field are felt immediately at an infinite distance from the source of disturbance. To overcome these deficiencies generalized thermoelasticity theories were developed consisting of a hyperbolic heat equation, which admit a finite speed for thermal signals. The first generalization was due to Lord and Shulman (1967), who obtained a wave-type heat equation by postulating a new law of heat conduction to replace the classical Fourier law. The second generalization is known as the theory of thermoelasticity with two relaxation times or the theory of temperature-rate-dependent thermoelasticity. Green and Lindsay (1972) obtained an explicit version of the constitutive equations later.

Nowacki (1966) and Eringen (1970) extended the linear theory of micropolar continua to include the thermal effect and formulated the micropolar thermoelasticity theory. One of the generalizations of the classical theory is the linear theory of elastic materials with stretch. A micropolar elastic solid with stretch which included the effect of axial stretch during the rotation of molecules was developed by Eringen (1971).

\footnotetext{
* To whom correspondence should be addressed
} 
Microstretch solids are capable of stretching and contracting independent of their translation and rotation. In these solids, the motion is characterized by seven degrees of freedom, namely three for translation, three for rotation and one for stretch. Examples of such elastic solids are porous media whose pores are filled with gas inviscid liquid, asphalt and composite fibrous materials. Eringen (1990) also developed a continuum theory of thermo-microstretch elastic solids. Green and Naghdi (1993) proposed the theory of thermoelasticity without energy dissipation and presented the derivation of a complete set of governing equations of the linearized version of the theory for homogeneous and isotropic materials in terms of displacement and temperature fields and proved the uniqueness of the solution of the corresponding initial mixed boundary value problem. Kumar and Deswal (2001) studied the disturbances caused by mechanical and thermal sources in a homogenous, isotropic generalized thermo-microstretch elastic medium by using the Laplace and Hankel transforms. Kumar et al. (2003) contributed to this field by studying a plane strain problem in a microstretch elastic solid. A problem of bending of microstretch elastic plates was investigated by Ciarletta (1999). Kumar and Partap (2009) investigated the propagation of axisymmetric free vibrations in a microstretch thermoelastic homogeneous isotropic solid which was subjected to stress free thermally insulated and isothermal conditions. Othman and Lotfy (2010) used a general model of the equations of generalized thermo-microstretch theories of Lord-Shulman (L-S), Green-Lindsay and classical dynamical coupled theory (C-D) for a homogenous isotropic elastic half space and compared results in the presence and absence of microstretch effect. Lotfy and Othman (2012) studied the effect of rotation of generalized thermomicrostretch elastic half space whose surface was subjected to Mode-I crack problem and compared the results for L-S and CD theories. Abbas and Othman (2012) used the finite element method to study the propagation of plane waves in a thermo-microstretch elastic solid half-space for the L-S and C-D model of field equations. Othman et al. (2013) studied the effect of the magnetic field on generalized thermomicrostretch isotropic elastic half space solid under rotation which is subjected to a mode-I crack problem in the context of G-N theory with the help of normal mode analysis.

\section{Formulation and solution of the problem}

We take a Cartesian coordinate system $\left(x_{1}, x_{2}, x_{3}\right)$ and the $x_{3}$-axis pointing vertically into the medium. Following Eringen (1990), Lord and Shulman (1967) and Green and Lindsay (1972) field equations and the constitutive relations without body load, body couples, heat sources and stretch force for a homogeneous, isotropic generalized thermo-microstretch elastic solid can be written as

$$
\begin{aligned}
& \lambda_{0} \nabla \phi^{*}+(\lambda+2 \mu+K) \nabla(\nabla \cdot \boldsymbol{u})-(\mu+K) \nabla \times \nabla \times \boldsymbol{u}+ \\
& +K \nabla \times \phi-v\left(1+\tau \frac{\partial}{\partial t}\right) \nabla T=\rho \frac{\partial^{2} \boldsymbol{u}}{\partial t^{2}}, \\
& (\alpha+\beta+\gamma) \nabla(\nabla \cdot \boldsymbol{\phi})-\gamma \nabla \times \nabla \times \phi+K \nabla \times \boldsymbol{u}-2 K \boldsymbol{\phi}=\rho J \frac{\partial^{2} \boldsymbol{u}}{\partial t^{2}}, \\
& \rho C^{*}\left(\frac{\partial T}{\partial t}+\tau_{0} \frac{\partial^{2} T}{\partial t^{2}}\right)+v T_{0}\left(\frac{\partial}{\partial t}+\Xi \tau \frac{\partial^{2}}{\partial t^{2}}\right) \nabla \cdot \boldsymbol{u}+v_{1} T_{0} \frac{\partial \phi^{*}}{\partial t}=K^{*} \nabla^{2} T, \\
& \alpha_{0} \nabla^{2} \phi^{*}+\frac{1}{3} v_{l} \phi^{*}-\frac{\lambda_{0}}{3} \nabla \cdot \boldsymbol{u}=\frac{3}{2} \rho J \frac{\partial^{2} \phi^{*}}{\partial t^{2}},
\end{aligned}
$$




$$
\begin{aligned}
& t_{i j}=\lambda u_{r, r} \delta_{i j}+\mu\left(u_{i, j}+u_{j, i}\right)+K\left(u_{j, i}-\epsilon_{i j r} \phi_{r}\right)-\left(T+\tau_{0} \frac{\partial T}{\partial t}\right) \delta_{i j} \\
& m_{i j}=\alpha \phi_{r, r} \delta_{i j}+\beta \phi_{i, j}+\gamma \phi_{j . i}, \\
& \lambda_{i}=\alpha_{0} \phi_{j, i}{ }^{*}
\end{aligned}
$$

where

$$
v=(3 \lambda+2 \mu+K) \alpha_{t_{1}}, \quad v_{1}=(3 \lambda+2 \mu+K) \alpha_{t_{2}}
$$

Since we are considering a two-dimensional plane strain problem, so we assume the components of the displacement vector $\boldsymbol{u}$ and microrotation vector $\phi$ are of the form

$$
\boldsymbol{u}=\left(u_{1}, 0, u_{3}\right), \quad \phi=\left(0, \phi_{2}, 0\right)
$$

Here due to symmetry about the $x_{3}$-axis all the quantities are independent of $x_{2}$, so that $\frac{\partial}{\partial x_{2}} \equiv 0$. With these considerations and using Eq.(2.8), the system of Eqs (2.1) to (2.7) reduces to

$$
\begin{aligned}
& (\lambda+\mu)\left[\frac{\partial^{2} u_{1}}{\partial x_{1}^{2}}+\frac{\partial^{2} u_{3}}{\partial x_{1} \partial x_{3}}\right]+(\mu+K)\left[\frac{\partial^{2} u_{1}}{\partial x_{1}^{2}}+\frac{\partial^{2} u_{1}}{\partial x_{3}^{2}}\right]-K \frac{\partial \phi_{2}}{\partial x_{3}}-v\left(1+\tau_{1} \frac{\partial}{\partial t}\right) \frac{\partial T}{\partial x_{1}}+\lambda_{0} \frac{\partial \phi^{*}}{\partial x_{1}}=\rho \frac{\partial^{2} u_{1}}{\partial t^{2}}, \\
& (\lambda+\mu)\left[\frac{\partial^{2} u_{1}}{\partial x_{3}^{2}}+\frac{\partial^{2} u_{3}}{\partial x_{3} \partial x_{1}}\right]+(\mu+K)\left[\frac{\partial^{2} u_{1}}{\partial x_{1}^{2}}+\frac{\partial^{2} u_{3}}{\partial x_{3}^{2}}\right]-K \frac{\partial \phi_{2}}{\partial x_{1}}-v\left(1+\tau_{1} \frac{\partial}{\partial t}\right) \frac{\partial T}{\partial x_{3}}+\lambda_{0} \frac{\partial \phi^{*}}{\partial x_{3}}=\rho \frac{\partial^{2} u_{3}}{\partial t^{2}}, \\
& \gamma\left[\frac{\partial^{2} \phi_{2}}{\partial x_{1}^{2}}+\frac{\partial^{2} \phi_{2}}{\partial x_{3}^{2}}\right]-2 K \phi_{2}+K\left[\frac{\partial u_{1}}{\partial x_{3}}-\frac{\partial u_{3}}{\partial x_{1}}\right]-K \frac{\partial \phi_{2}}{\partial x_{3}}=\rho J \frac{\partial^{2} \phi_{2}}{\partial t^{2}}, \\
& K^{*}\left[\frac{\partial^{2} T}{\partial x_{1}^{2}}+\frac{\partial^{2} T}{\partial x_{3}^{2}}\right]=\rho C^{*}\left[\frac{\partial T}{\partial t}+\tau_{0} \frac{\partial^{2} T}{\partial t^{2}}\right]+v T_{0}\left[\frac{\partial}{\partial t}+\Xi \tau_{0} \frac{\partial^{2}}{\partial t^{2}}\right]\left(\frac{\partial u_{1}}{\partial x_{1}}+\frac{\partial u_{3}}{\partial x_{3}}\right)+v T_{0} \frac{\partial \phi^{*}}{\partial t}, \\
& \alpha_{0}\left[\frac{\partial^{2} \phi^{*}}{\partial x_{1}^{2}}+\frac{\partial^{2} \phi^{*}}{\partial x_{3}^{2}}\right]+\frac{1}{3} v_{1} T-\frac{1}{3} \lambda_{1} \phi^{*}-\frac{1}{3} \lambda_{0}\left(\frac{\partial u_{1}}{\partial x_{1}}+\frac{\partial u_{3}}{\partial x_{3}}\right)=\frac{3}{2} \rho J \frac{\partial^{2} \phi^{*}}{\partial t^{2}},
\end{aligned}
$$

where

$$
\nabla^{2}=\frac{\partial^{2}}{\partial x_{1}^{2}}+\frac{\partial^{2}}{\partial x_{3}^{2}}
$$

We define the dimensionless quantities as 


$$
\begin{aligned}
& x_{1}^{\prime}=\frac{\omega^{*} x_{1}}{C_{1}}, \quad x_{3}^{\prime}=\frac{\omega^{*} x_{3}}{C_{1}}, \quad t^{\prime}=\omega^{*} t, \quad \tau_{0}^{\prime}=\omega^{*} \tau_{0}, \quad T^{\prime}=\frac{T}{T_{0}}, \quad u_{1}^{\prime}=\frac{\rho \omega^{*} C_{l} u_{1}}{v T_{0}}, \\
& u_{3}^{\prime}=\frac{\rho \omega^{*} C_{1} u_{3}}{v T_{0}}, \quad \phi_{2}^{\prime}=\frac{\rho C_{1}^{2}}{v T_{0}} \phi_{2}, \quad t_{33}^{\prime}=\frac{t_{33}}{v T_{0}}, \quad t_{31}^{\prime}=\frac{t_{31}}{v T_{0}}, \quad m_{32}^{\prime}=\frac{\omega^{*}}{C_{1} v T_{0}} m_{32}, \\
& \omega^{*}=\frac{C^{*}(\lambda+2 \mu)}{K^{*}}, \quad C_{1}^{2}=\frac{\lambda+2 \mu}{\rho}, \quad \phi^{*^{\prime}}=\frac{\rho c_{1}^{2}}{v T_{0}} \phi^{*}, \quad \lambda_{3}^{\prime}=\frac{\omega^{*}}{c_{1} v T_{0}} \lambda_{3}, \quad \tau_{1}^{\prime}=\omega^{*} \tau_{1} .
\end{aligned}
$$

Using the dimensionless quantities defined in Eqs (2.14), the system of Eqs (2.9)-(2.13) may be recast into a dimensionless form after suppressing the primes as

$$
\begin{aligned}
& m_{1} \frac{\partial^{2} u_{1}}{\partial x_{1}^{2}}+m_{2} \frac{\partial^{2} u_{3}}{\partial x_{1} \partial x_{3}}+m_{3} \frac{\partial^{2} u_{1}}{\partial x_{3}^{2}}-m_{4} \frac{\partial \phi_{2}}{\partial x_{3}}-\left(1+\tau_{1} \frac{\partial}{\partial t}\right) \frac{\partial T}{\partial x_{1}}+m_{5} \frac{\partial \phi^{*}}{\partial x_{1}}=\frac{\partial^{2} u_{1}}{\partial t^{2}} \\
& m_{1} \frac{\partial^{2} u_{3}}{\partial x_{3}^{2}}+m_{2} \frac{\partial^{2} u_{3}}{\partial x_{1} \partial x_{3}}+m_{3} \frac{\partial^{2} u_{3}}{\partial x_{1}^{2}}+m_{4} \frac{\partial \phi_{2}}{\partial x_{1}}-\left(1+\tau_{1} \frac{\partial}{\partial t}\right) \frac{\partial T}{\partial x_{3}}+m_{5} \frac{\partial \phi^{*}}{\partial x_{3}}=\frac{\partial^{2} u_{3}}{\partial t^{2}} \\
& {\left[\frac{\partial^{2} \phi_{2}}{\partial x_{1}^{2}}+\frac{\partial^{2} \phi_{2}}{\partial x_{3}^{2}}\right]-2 m_{6} \phi_{2}+m_{6}\left[\frac{\partial u_{1}}{\partial x_{3}}-\frac{\partial u_{3}}{\partial x_{1}}\right]=m_{7} \frac{\partial^{2} \phi_{2}}{\partial t^{2}},} \\
& {\left[\frac{\partial^{2} T}{\partial x_{1}^{2}}+\frac{\partial^{2} T}{\partial x_{3}^{2}}\right]-\left[\frac{\partial T}{\partial t}+\tau_{0} \frac{\partial^{2} T}{\partial t^{2}}\right]=\varepsilon\left[\frac{\partial}{\partial t}\left(\frac{\partial u_{1}}{\partial x_{1}}+\frac{\partial u_{3}}{\partial x_{1}}\right)+\Xi \tau_{0} \frac{\partial^{2}}{\partial t^{2}}\left(\frac{\partial u_{1}}{\partial x_{1}}+\frac{\partial u_{3}}{\partial x_{1}}\right)\right]+} \\
& +\varepsilon m_{8} \frac{\partial \phi^{*}}{\partial t}, \\
& \frac{\partial^{2} \phi^{*}}{\partial x_{1}^{2}}++\frac{\partial^{2} \phi^{*}}{\partial x_{3}^{2}}+T m_{9}-m_{10} \phi^{*}-m_{11}\left(\frac{\partial u_{1}}{\partial x_{1}}+\frac{\partial u_{3}}{\partial x_{1}}\right)=m_{12} \frac{\partial^{2} \phi^{*}}{\partial t^{2}},
\end{aligned}
$$

where

$$
\begin{aligned}
& m_{1}=\frac{\lambda+2 \mu+K}{\rho C_{1}^{2}}, \quad m_{2}=\frac{\lambda+\mu}{\rho C_{1}^{2}}, \quad m_{3}=\frac{\mu+K}{\rho C_{1}^{2}}, \quad m_{4}=\frac{K}{\rho C_{1}^{2}}, \quad m_{5}=\frac{\lambda_{0}}{\rho C_{1}^{2}}, \\
& m_{6}=\frac{K C_{1}^{2}}{\gamma \omega^{* 2}}, \quad m_{7}=\frac{\rho J C_{1}^{2}}{\gamma}, \quad m_{8}=\frac{v_{1}}{v}, \quad m_{9}=\frac{\rho v_{1} c_{1}^{4}}{3 v \alpha_{0} \omega^{* 2}}, \quad m_{10}=\frac{\lambda_{1} c_{1}^{2}}{3 \alpha_{0} \omega^{* 2}}, \\
& m_{11}=\frac{\lambda_{0} c_{1}^{2}}{3 \alpha_{0} \omega^{* 2}}, \quad m_{12}=\frac{3 \rho J c_{1}^{2}}{2 \alpha_{0}}
\end{aligned}
$$

Applying the Laplace and Fourier transforms to Eqs (2.15) - (2.19) defined by 


$$
\begin{aligned}
& \bar{f}\left(x_{1}, x_{3}, p\right)=L\left\{f\left(x_{1}, x_{3}, t\right)\right\}=\int_{0}^{\infty} f\left(x_{1}, x_{3}, t\right) \exp (-p t) d t, \\
& \tilde{f}\left(\xi, x_{3}, p\right)=F\left\{\bar{f}\left(x_{1}, x_{3}, p\right)\right\}=\int_{-\infty}^{\infty} \mathrm{e}^{-1 x_{1} \xi} \bar{f}\left(x_{1}, x_{3}, p\right) d x_{1},
\end{aligned}
$$

we obtain

$$
\begin{aligned}
& D^{2} \tilde{u}_{1}=\frac{1}{m_{3}}\left\{\left(m_{1} \xi^{2}+p^{2}\right) \tilde{u}_{1}-i \xi m_{2} D \tilde{u}_{3}+m_{4} D \tilde{\phi}_{2}+i \xi\left(1+\tau_{1} p\right) \tilde{T}-i \xi m_{5} \tilde{\phi}^{*}\right\}, \\
& D^{2} \tilde{u}_{3}=\frac{1}{m_{1}}\left\{\left(m_{3} \xi^{2}+p^{2}\right) \tilde{u}_{3}-i \xi m_{2} D \tilde{u}_{1}-i \xi m_{4} \tilde{\phi}_{2}+\left(1+\tau_{1} p\right) D \tilde{T}-m_{5} D \tilde{\phi}^{*}\right\}, \\
& D^{2} \tilde{\phi}_{2}=-m_{6} D \tilde{u}_{1}+\xi m_{6} \tilde{u}_{3}+\left(2 m_{6}+\xi^{2}+m_{7} p^{2}\right) \tilde{\phi}_{2}, \\
& D^{2} \tilde{T}=\operatorname{cip}\left(1+\tau_{0} p \Xi\right) \xi \tilde{u}_{1}+\varepsilon p\left(1+\tau_{0} p \Xi\right) D \tilde{u}_{3}+\left\{\xi^{2}+p\left(1+\tau_{0} p\right)\right\} \tilde{T}+\varepsilon p m_{8} \tilde{\phi}^{*}, \\
& D^{2} \tilde{\phi}^{*}=\xi m_{11} \tilde{u}_{1}+m_{11} D \tilde{u}_{3}-m_{9} \tilde{T}+\left(m_{12} p^{2}+\xi^{2}+m_{10}\right) \tilde{\phi}^{*} .
\end{aligned}
$$

The system of Eqs (2.22) - (2.26) can be written as

$$
\frac{d W\left(\xi, x_{3}, p\right)}{d x_{3}}=A(\xi, p) W\left(\xi, x_{3}, p\right)
$$

where

$$
\begin{aligned}
& W=\left[\begin{array}{c}
U \\
U^{\prime}
\end{array}\right], \quad A=\left[\begin{array}{cc}
O & I \\
A_{2} & A_{1}
\end{array}\right], \quad U=\left[\begin{array}{lllll}
\tilde{u}_{1} & \tilde{u}_{3} & \tilde{\phi}_{2} & \tilde{T} & \tilde{\phi}^{*}
\end{array}\right]^{t}, \\
& A_{1}=\left[\begin{array}{ccccc}
0 & -\frac{i \xi m_{2}}{m_{3}} & \frac{m_{4}}{m_{3}} & 0 & 0 \\
-\frac{i \xi m_{2}}{m_{1}} & 0 & 0 & \frac{1+\tau_{1} p}{m_{1}} & -\frac{m_{5}}{m_{1}} \\
-m_{6} & 0 & 0 & 0 & 0 \\
0 & \varepsilon p\left(1+\tau_{0} p \Xi\right) & 0 & 0 & 0 \\
0 & m_{11} & 0 & 0 & 0
\end{array}\right],
\end{aligned}
$$




$$
A_{2}=\left[\begin{array}{ccccc}
\frac{\left(m_{1} \xi^{2}+p^{2}\right)}{m_{3}} & 0 & 0 & \frac{i \xi\left(1+\tau_{1} p\right)}{m_{3}} & -\frac{i m_{5} \xi}{m_{3}} \\
0 & \frac{\left(m_{3} \xi^{2}+p^{2}\right)}{m_{1}} & -\frac{i m_{4} \xi}{m_{1}} & 0 & 0 \\
0 & i m_{6} \xi & \left(2 m_{6}+\xi^{2}+m_{7} p^{2}\right) & 0 & 0 \\
\varepsilon p\left(1+\tau_{0} p \Xi\right) \xi & 0 & 0 & \xi^{2}+p\left(1+\tau_{0} p\right) & \varepsilon p m_{8} \\
i \xi m_{11} & 0 & 0 & -m_{9} & \left(m_{12} p^{2}+\xi^{2}+m_{10}\right)
\end{array}\right],
$$

where $\mathrm{O}$ is the null matrix, I unit matrix of order 4,[] $]^{t}$ transpose of matrix [] and $D=\frac{d}{d x_{3}}$.

To solve Eq.(2.27), we take

$$
W\left(\xi, x_{3}, p\right)=W(\xi, p) e^{q x_{3}},
$$

for some parameter $q$ so that

$$
A(\xi, p) W\left(\xi, x_{3}, p\right)=q W\left(\xi, x_{3}, p\right),
$$

which leads to the eigen value problem. The characteristic equation corresponding to the matrix $\mathrm{A}$ is given by

$$
\operatorname{det}(A-q I)=0,
$$

On expanding (2.30), we get

$$
q^{10}-\sigma_{1} q^{8}+\sigma_{2} q^{6}-\sigma_{3} q^{4}+\sigma_{4} q^{2}+\sigma_{5}=0
$$

where $\sigma_{i}{ }^{\prime} s(i=1,2, . ., 5)$ are functions of $\xi$ and $p$.

The eigen values of the matrix A are characteristic roots of Eq.(2.31). The eigen vectors $X(\xi, p)$ corresponding to the eigen value $q_{s}$ can be determined by solving the system of homogenous equations

$$
[A-q I] X(\xi, p)=0 .
$$

The set of eigen vectors $X_{s}(\xi, p) ; s=1,2 \ldots 10$ is be obtained as

$$
X_{s}(\xi, p)=\left[\begin{array}{c}
X_{s 1}(\xi, p) \\
X_{s 2}(\xi, p)
\end{array}\right]
$$

where 
$X_{s 1}=\left[\begin{array}{lllll}a_{s} q_{s} & b_{s} & -\xi & c_{s} & d_{s}\end{array}\right]^{t}, \quad X_{s 2}=\left[\begin{array}{lllll}a_{s} q_{s}^{2} & b_{s} q_{s} & -\xi q_{s} & c_{s} q_{s} & d_{s} q_{s}\end{array}\right]^{t}$, $q=q_{s} ; \quad s=1,2,3,4,5$,

$X_{l 1}=\left[\begin{array}{lllll}-a_{s} q_{s} & b_{s} & -\xi & c_{s} & d_{s}\end{array}\right]^{t}, \quad X_{l 2}=\left[\begin{array}{lllll}a_{s} q_{s}^{2} & -b_{s} q_{s} & \xi q_{s} & -c_{s} q_{s} & -d_{s} q_{s}\end{array}\right]^{t}$ $l=s+5, \quad q=-q_{s}$,

for $s=1,2,3,4,5$,

$a_{s}=\frac{q_{s}}{\Delta_{s}}\left(m_{6} g_{11} F_{3}+F_{2} H_{3}\right), \quad b_{s}=\frac{i}{\Delta_{s}}\left(m_{6} g_{11} F_{3} q_{s}^{2}+F_{1} H_{3}\right)$,

$\Delta_{s}=m_{6}\left(F_{1}-F_{2} q_{s}^{2}\right), \quad c_{s}=\frac{q_{s}\left[\varepsilon p m_{8}\left(a_{s} H_{1}+\xi b_{s} g_{10}-\xi g_{11}\right)-\xi g_{7} g_{9}\left(b_{s}+i \xi a_{s}\right)\right]}{\xi\left(\varepsilon p m_{8} g_{6}+g_{7} H_{4}\right)}$, $d_{s}=\frac{\left[c_{s} m_{9}-m_{11} q_{s}\left(b_{s}+i \xi a_{s}\right)\right]}{H_{5}}$,

$F_{1}=a^{*} k H_{1}-b^{*} k \xi^{2}+b^{*} f \xi^{2} H_{5}+g H_{1} H_{2} H_{3}-a^{*} f h \xi^{2}-g h \xi^{2} H_{4}$, $F_{2}=a^{*} k c^{*}-b^{*} k+b^{*} f H_{5}+g c^{*} H_{4} H_{5}-a^{*} f h-g h H_{4}, \quad F_{3}=a^{*} k+g H_{4} H_{5}$, $H_{1}=g_{1}-q^{2}, \quad H_{2}=g_{2}-q^{2}, \quad H_{3}=g_{3}-q^{2}, \quad H_{4}=g_{4}-q^{2}$, $H_{5}=g_{5}-q^{2}, \quad a^{*}=\in m_{8}, \quad b^{*}=g_{7} g_{9}, \quad c^{*}=g_{10}, \quad f=g_{6}, \quad g=g_{7}$, $h=g_{7} m_{11}, \quad k=g_{7} m_{9}, \quad f_{1}=\frac{m_{1} \xi^{2}+p^{2}}{m_{3}}, \quad f_{2}=\frac{m_{2} \xi^{2}+p^{2}}{m_{1}}$, $f_{3}=2 m_{6}+\xi^{2}+m_{7} p^{2}, \quad f_{4}=m_{10}+\xi^{2}+m_{12} p^{2}, \quad f_{5}=p+\xi^{2}+\tau_{0} p^{2}$, $f_{6}=f_{1} f_{2}, \quad f_{7}=f_{1} f_{3}, \quad f_{8}=f_{1} f_{4}, \quad f_{9}=f_{1} f_{5}, \quad f_{10}=f_{2} f_{3}$, $f_{11}=f_{2} f_{4}, \quad f_{12}=f_{2} f_{5}, \quad f_{13}=f_{3} f_{4}, \quad f_{14}=f_{3} f_{5}$, $f_{15}=f_{4} f_{5}, \quad g_{1}=\sum_{l=1}^{5} f_{l}, \quad g_{2}=\sum_{l=6}^{15} f_{l}, \quad g_{3}=\sum_{l=16}^{25} f_{l}, \quad g_{4}=\sum_{l=26}^{30} f_{l}$, 


$$
\begin{aligned}
& g_{5}=f_{1} f_{2} f_{3} f_{4} f_{5}, \quad g_{6}=\frac{\varepsilon p\left(1+p \tau_{0}\right)}{m_{1}}, \quad g_{7}=\frac{m_{5} m_{11}}{m_{1} m_{3}}, \\
& g_{8}=\xi^{2}\left(m_{2}-m_{1}+m_{3}\right) g_{7}, \quad g_{9}=\xi^{2}\left(f_{1}+f_{3}+f_{5}+m_{4} m_{6}\right) g_{7}, \\
& g_{10}=\frac{m_{4} m_{6}\left(1-g_{6}\right)}{m_{3}}, \quad g_{11}=\frac{\xi^{2}\left(m_{2}-m_{1}\right) g_{6}}{m_{3}} .
\end{aligned}
$$

Thus the solution of Eq.(2.27) as given by Singh et al. (2011) is

$$
W\left(\xi, x_{3}, q\right)=\sum_{s=1}^{5}\left[B_{s} X_{s} \exp \left(q_{s} x_{3}\right)+B_{s+5} X_{s+5} \exp \left(-q_{S} x_{3}\right)\right]
$$

where $B_{s}{ }^{\prime} s(s=1,2, \ldots, 10)$ are arbitrary constants. Equation (2.34) represents the solution of the generalized thermo-microstretch elastic medium for the plane strain case and gives displacement, microrotation, temperature distribution and scalar microstretch in the transformed domain.

\section{Application}

We consider an infinite generalized thermo-microstretch elastic space in which a concentrated force $F=-F_{0} \delta\left(x_{1}\right) \delta(t)$ where $F_{0}$ is the magnitude of the force, acting in the direction of the $x_{3}$-axis at the origin of the Cartesian co-ordinate system as shown in Fig.1.

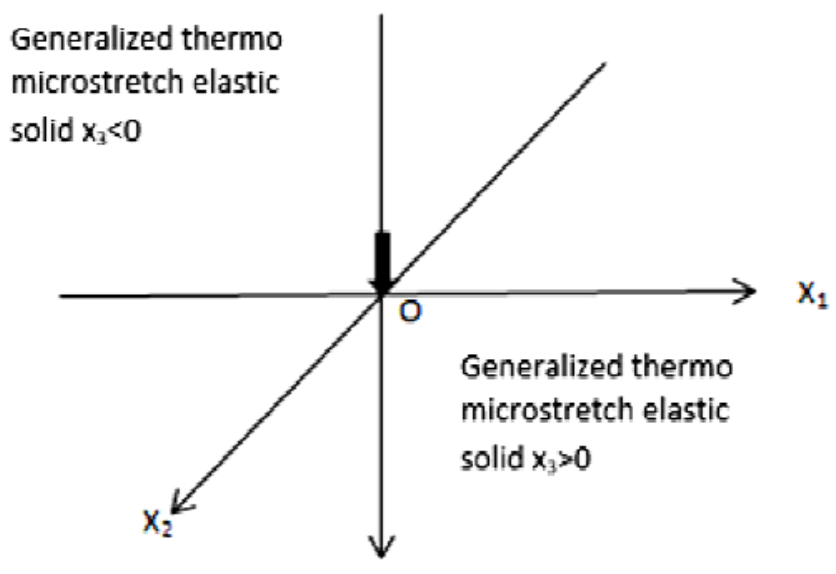

The boundary conditions for the plane $x_{3}=0$ are given by

$$
\begin{aligned}
& u_{1}\left(x_{1}, 0^{+}, t\right)-u_{1}\left(x_{1}, 0^{-}, t\right)=0, \quad u_{3}\left(x_{1}, 0^{+}, t\right)-u_{3}\left(x_{1}, 0^{-}, t\right)=0, \\
& \phi_{2}\left(x_{1}, 0^{+}, t\right)-\phi_{2}\left(x_{1}, 0^{-}, t\right)=0, \phi^{*}\left(x_{1}, 0^{+}, t\right)-\phi^{*}\left(x_{1}, 0^{-}, t\right)=0,
\end{aligned}
$$




$$
\begin{array}{ll}
T\left(x_{1}, 0^{+}, t\right)-u_{1}\left(x_{1}, 0^{-}, t\right)=0, & \frac{\partial T}{\partial \mathrm{x}_{3}}\left(x_{1}, 0^{+}, t\right)-\frac{\partial T}{\partial x_{3}}\left(x_{1}, 0^{-}, t\right)=0, \\
t_{31}\left(x_{1}, 0^{+}, t\right)-t_{31}\left(x_{1}, 0^{-}, t\right)=0, & t_{33}\left(x_{1}, 0^{+}, t\right)-t_{33}\left(x_{1}, 0^{-}, t\right)=-F_{0} \delta\left(x_{1}\right) \delta(t), \\
m_{32}\left(x_{1}, 0^{+}, t\right)-m_{32}\left(x_{1}, 0^{-}, t\right)=0, & \lambda_{3}\left(x_{1}, 0^{+}, t\right)-\lambda_{3}\left(x_{1}, 0^{-}, t\right)=0 .
\end{array}
$$

For $\boldsymbol{x}_{3}>\mathbf{0}$ : Making use of Eqs (2.8) and (2.14) on Eqs (2.5) - (2.7) and $F_{0}^{\prime}=\frac{F_{0}}{K}$, we get the stresses in the non-dimensional form with primes. After suppressing the primes and applying the Laplace and Fourier transforms defined by Eqs (2.20) and (2.21) on the resulting equations and using boundary conditions (3.1)(3.5), we get the transformed components of displacement, microrotation, scalar microstretch, temperature distribution, tangential force stress, normal force stress, tangential couple stress and microstress for $x_{3}>0$, given by

$$
\begin{aligned}
& \tilde{u}_{1}\left(\xi, x_{3}, p\right)=-\sum_{s=1}^{5} a_{s} q_{s} B_{s+5} e^{-q_{s} x_{3}} \\
& \tilde{u}_{3}\left(\xi, x_{3}, p\right)=\sum_{s=1}^{5} b_{s} B_{s+5} e^{-q_{s} x_{3}} \\
& \tilde{\phi}_{2}\left(\xi, x_{3}, p\right)=-\xi \sum_{s=1}^{5} B_{s+5} e^{-q_{s} x_{3}}, \\
& \tilde{\phi}^{*}\left(\xi, x_{3}, p\right)=\sum_{s=1}^{5} c_{s} B_{s+5} e^{-q_{s} x_{3}} \\
& \tilde{T}\left(\xi, x_{3}, p\right)=\sum_{s=1}^{5} d_{s} B_{s+5} e^{-q_{s} x_{3}}, \\
& \tilde{t}_{31}\left(\xi, x_{3}, p\right)=\sum_{s=1}^{5}\left(m_{3} a_{s} q_{s}^{2}+i \xi b_{s} m_{14}+\xi m_{4}\right) B_{s+5} e^{-q_{s} x_{3}}, \\
& \tilde{t}_{33}\left(\xi, x_{3}, p\right)=\sum_{s=1}^{5}\left[i \xi m_{15} a_{s} q_{s}+m_{1} b_{s} q_{s}+m_{15} c_{s}+\left(1+\tau_{0} p\right) d_{s}\right] B_{s+5} e^{-q_{s} x_{3}}, \\
& \tilde{m}_{32}\left(\xi, x_{3}, p\right)=\xi m_{13} \sum_{s=1}^{5} q_{s} B_{s+5} e^{-q_{s} x_{3}},
\end{aligned}
$$




$$
\tilde{\lambda}_{3}\left(\xi, x_{3}, p\right)=-m_{16} \sum_{s=1}^{5} c_{s} q_{s} B_{s+5} e^{-q_{s} x_{3}}
$$

For $\boldsymbol{x}_{3}<\boldsymbol{0}$ : the above expressions get suitably modified, e.g.,

$$
\tilde{u}_{1}\left(\xi, x_{3}, p\right)=\sum_{s=1}^{5} a_{s} q_{s} B_{s} e^{q_{s} x_{3}}
$$

where

$$
m_{13}=\frac{\gamma \omega^{* 2}}{\rho C_{1}^{4}}, \quad m_{14}=\frac{\mu}{\rho C_{1}^{2}}, \quad m_{15}=\frac{\lambda}{\rho C_{1}^{2}}, \quad m_{16}=\frac{\alpha \omega^{* 2}}{\rho C_{1}^{4}} .
$$

Making use of the transformed displacement, microrotation, temperature distribution, scalar microstretch and stress components given by Eqs (3.6)-(3.14) in region $x_{3}>0$ and equations for the region $x_{3}<0$ in the boundary conditions, we obtain ten linear relations between $B_{s}{ }^{\prime} s(s=1,2, . ., 10)$ which on solving give

$$
\begin{aligned}
& B_{1}=B_{6}=\frac{F_{0}}{2 q_{1} \Delta_{1}^{*}}\left[\left(a_{3}-a_{1}\right)\left\{\left(c_{4}-c_{2}\right)\left(d_{5}-d_{2}\right)-\left(d_{4}-d_{2}\right)\left(c_{5}-c_{2}\right)\right\}+\right. \\
& +\left(a_{4}-a_{2}\right)\left\{\left(c_{1}-c_{2}\right)\left(d_{3}-d_{2}\right)-\left(d_{5}-d_{2}\right)\left(c_{3}-c_{2}\right)\right\}+ \\
& \left.+\left(a_{5}-a_{2}\right)\left\{\left(c_{3}-c_{2}\right)\left(d_{4}-d_{2}\right)-\left(d_{3}-d_{2}\right)\left(c_{4}-c_{2}\right)\right\}\right], \\
& B_{2}=B_{7}=\frac{F_{0}}{2 q_{2} \Delta_{1}^{*}}\left[\left(a_{3}-a_{1}\right)\left\{\left(c_{5}-c_{1}\right)\left(d_{4}-d_{1}\right)-\left(d_{5}-d_{1}\right)\left(c_{4}-c_{1}\right)\right\}+\right. \\
& +\left(a_{4}-a_{1}\right)\left\{\left(c_{3}-c_{1}\right)\left(d_{5}-d_{1}\right)-\left(d_{3}-d_{1}\right)\left(c_{5}-c_{1}\right)\right\}+ \\
& \left.+\left(a_{5}-a_{1}\right)\left\{\left(c_{4}-c_{1}\right)\left(d_{3}-d_{1}\right)-\left(d_{4}-d_{1}\right)\left(c_{3}-c_{1}\right)\right\}\right], \\
& B_{3}=B_{8}=\frac{F_{0}}{2 q_{3} \Delta_{1}^{*}}\left[\left(a_{2}-a_{1}\right)\left\{\left(c_{5}-c_{1}\right)\left(d_{5}-d_{1}\right)-\left(d_{4}-d_{1}\right)\left(c_{5}-c_{1}\right)\right\}+\right. \\
& +\left(a_{4}-a_{1}\right)\left\{\left(c_{5}-c_{1}\right)\left(d_{2}-d_{1}\right)-\left(d_{5}-d_{1}\right)\left(c_{2}-c_{1}\right)\right\}+ \\
& \left.+\left(a_{5}-a_{1}\right)\left\{\left(c_{2}-c_{1}\right)\left(d_{4}-d_{1}\right)-\left(d_{2}-d_{1}\right)\left(c_{4}-c_{1}\right)\right\}\right], \\
& +\left(a_{3}-a_{1}\right)\left\{\left(c_{2}-c_{1}\right)\left(d_{5}-d_{1}\right)-\left(c_{5}-c_{1}\right)\left(d_{2}-d_{1}\right)\right\}+ \\
& \left.+\left(a_{5}-a_{1}\right)\left\{\left(c_{3}-c_{1}\right)\left(d_{2}-d_{1}\right)-\left(c_{2}-c_{1}\right)\left(d_{3}-d_{1}\right)\right\}\right],
\end{aligned}
$$




$$
\begin{aligned}
& B_{5}=B_{10}=\frac{F_{0}}{2 q_{5} \Delta_{1}^{*}}\left[\left(a_{2}-a_{1}\right)\left\{\left(c_{3}-c_{1}\right)\left(d_{4}-d_{1}\right)-\left(d_{3}-d_{1}\right)\left(c_{4}-c_{1}\right)\right\}+\right. \\
& +\left(a_{3}-a_{1}\right)\left\{\left(c_{4}-c_{1}\right)\left(d_{2}-d_{1}\right)-\left(d_{4}-d_{1}\right)\left(c_{2}-c_{1}\right)\right\}+ \\
& \left.+\left(a_{4}-a_{1}\right)\left\{\left(c_{2}-c_{1}\right)\left(d_{3}-d_{1}\right)-\left(d_{2}-d_{1}\right)\left(c_{3}-c_{1}\right)\right\}\right]
\end{aligned}
$$

where

$$
\begin{aligned}
& \Delta_{1}^{*}=m_{1}\left[\left(c_{2}-c_{5}\right)\left\{a_{1}\left(d_{3} b_{4}-d_{4} b_{3}\right)+a_{3}\left(d_{4} b_{1}-d_{1} b_{4}\right)+a_{4}\left(d_{1} b_{3}-d_{3} b_{1}\right)\right\}+\right. \\
& +\left(c_{2}-c_{3}\right)\left\{a_{1}\left(d_{4} b_{5}-d_{5} b_{4}\right)+a_{4}\left(d_{5} b_{1}-d_{1} b_{5}\right)+a_{5}\left(d_{1} b_{4}-d_{4} b_{1}\right)\right\}+ \\
& +\left(c_{4}-c_{5}\right)\left\{a_{1}\left(d_{2} b_{3}-d_{3} b_{2}\right)+a_{2}\left(d_{3} b_{1}-d_{1} b_{3}\right)+a_{3}\left(d_{1} b_{2}-d_{2} b_{1}\right)\right\}+ \\
& +\left(c_{2}-c_{4}\right)\left\{a_{1}\left(d_{5} b_{3}-d_{3} b_{5}\right)+a_{3}\left(d_{1} b_{5}-d_{5} b_{1}\right)+a_{5}\left(d_{3} b_{1}-d_{1} b_{3}\right)\right\}+ \\
& +\left(c_{4}-c_{3}\right)\left\{a_{1}\left(d_{5} b_{2}-d_{2} b_{5}\right)+a_{2}\left(d_{1} b_{5}-d_{5} b_{1}\right)+a_{5}\left(d_{2} b_{1}-d_{1} b_{2}\right)\right\}+ \\
& +\left(c_{5}-c_{3}\right)\left\{a_{1}\left(d_{2} b_{4}-d_{4} b_{2}\right)+a_{4}\left(d_{1} b_{2}-d_{2} b_{1}\right)+a_{2}\left(d_{4} b_{1}-d_{1} b_{4}\right)\right\}+ \\
& +\left(c_{3}-c_{1}\right)\left\{a_{2}\left(d_{4} b_{5}-d_{5} b_{4}\right)+a_{4}\left(d_{5} b_{2}-d_{2} b_{5}\right)+a_{5}\left(d_{2} b_{4}-d_{4} b_{2}\right)\right\}+ \\
& +\left(c_{5}-c_{1}\right)\left\{a_{2}\left(d_{3} b_{4}-d_{4} b_{3}\right)+a_{3}\left(d_{4} b_{2}-d_{2} b_{4}\right)+a_{4}\left(d_{2} b_{3}-d_{3} b_{2}\right)\right\}+ \\
& +\left(c_{4}-c_{1}\right)\left\{a_{2}\left(d_{5} b_{3}-d_{3} b_{5}\right)+a_{3}\left(d_{2} b_{5}-d_{5} b_{2}\right)+a_{5}\left(d_{3} b_{2}-d_{2} b_{3}\right)\right\}+ \\
& +\left(c_{2}-c_{1}\right)\left\{a_{3}\left(d_{5} b_{4}-d_{4} b_{5}\right)+a_{4}\left(d_{3} b_{5}-d_{5} b_{3}\right)+a_{5}\left(d_{4} b_{3}-d_{3} b_{4}\right)\right\} .
\end{aligned}
$$

Thus functions $\tilde{u}_{1}, \tilde{u}_{3}, \tilde{\phi}_{2}, \tilde{T}, \tilde{t}_{31}, \tilde{t}_{33}, \tilde{m}_{32}, \tilde{\phi}^{*}$ and $\tilde{\lambda}_{3}$ have been determined in the transform domain and these enable us to find the displacements, microrotation, temperature distribution field, stresses, scalar microstretch and microstress.

\section{Method for the inversion of transforms}

To obtain the solution of the problem in the physical domain, we must invert the transforms for both theories that is L-S and G-L. These expressions are functions of $x_{3}$, the parameters of the Laplace and Fourier transforms $\mathrm{p}$ and $\xi$, respectively, and hence are of the form $\bar{f}\left(x_{1}, x_{3}, p\right)$. To get the function $\bar{f}\left(x_{1}, x_{3}, p\right)$ in the physical domain, first we invert the Fourier transform using

$$
\bar{f}\left(x_{1}, x_{3}, p\right)=\int_{-\infty}^{\infty} \exp \left(i \xi x_{1}\right) \tilde{f}\left(\xi, x_{3}, p\right) d \xi=\frac{1}{\pi} \int_{0}^{\infty}\left\{\cos \left(\xi x_{1}\right) \tilde{f}_{e}+i \sin \left(\xi x_{1}\right) \tilde{f}_{0}\right\} d \xi
$$

The last step in the inversion process is to evaluate the integral in Eq.(4.1). This was done using Romberg's integration with an adaptive step size. This method uses the results from successive refinements of the extended trapezoidal rule followed by extrapolation of the results to the limit when the step size tends to zero. The details can be found in Press et al. (1986). 


\section{Numerical results and discussion} crystal as

Following Eringen (1984), we take the following values of relevant parameters for magnesium

$$
\begin{aligned}
& \lambda=9.4 \times 10^{10} \mathrm{~N} / \mathrm{m}^{2}, \quad \mu=4 \times 10^{10} \mathrm{~N} / \mathrm{m}^{2}, \quad K=1 \times 10^{10} \mathrm{~N} / \mathrm{m}^{2}, \quad \rho=1.74 \times 10^{3} \mathrm{~kg} / \mathrm{m}^{3}, \\
& \gamma=0.779 \times 10^{-9} \mathrm{~N}, \quad J=0.2 \times 10^{-19} \mathrm{~m}^{2}, \quad K^{*}=1.1753 \times 10^{-19} \mathrm{~m}^{2}, \\
& \omega^{*}=0.0787 \times 10^{-1} \mathrm{~N} \mathrm{sec} / \mathrm{m}^{2}, \quad \tau_{0}=6.131 \times 10^{-13} \mathrm{~s}, \quad \tau_{1}=8.765 \times 10^{-13} \mathrm{~s}, \\
& \varepsilon=0.073, \quad T_{0}=296 \mathrm{~K}, \quad \lambda_{0}=0.5 \times 10^{10} \mathrm{~N} / \mathrm{m}^{2}, \quad \lambda_{1}=0.5 \times 10^{10} \mathrm{~N} / \mathrm{m}^{2}, \\
& \alpha_{0}=0.779 \times 10^{-9} \mathrm{~N}, \quad C^{*}=3.525 \mathrm{~J} \mathrm{Kg^{-1 } \mathrm { K } ^ { - 1 } .}
\end{aligned}
$$

The variations of the non-dimensional normal displacement $U_{3}\left(=2 u_{3} / F_{0}\right)$, non-dimensional normal stress $T_{33}\left(=2 t_{33} / F_{0}\right)$, non-dimensional tangential couple stress $M_{32}\left(=2 m_{32} / F_{0}\right)$, non-dimensional microstress $\lambda_{3}^{*}\left(=2 \lambda_{3} / F_{0}\right)$ and non-dimensional temperature distribution $T^{*}\left(=2 T / F_{0}\right)$ with nondimensional distance ' $x_{1}$ ' at the plane $x_{3}=1, h=10^{-10} \mathrm{~m}$ and coupling coefficient $\varepsilon=0.073$ have been shown in Figs 2 - 6 for (a) generalized thermo-microstretch elastic (GTMSE) solid (b) generalized thermo micropolar elastic (GTME) solid; (c) generalized thermoelastic (GTE) solid for time $t=0.1,0.125$ and 0.5 .

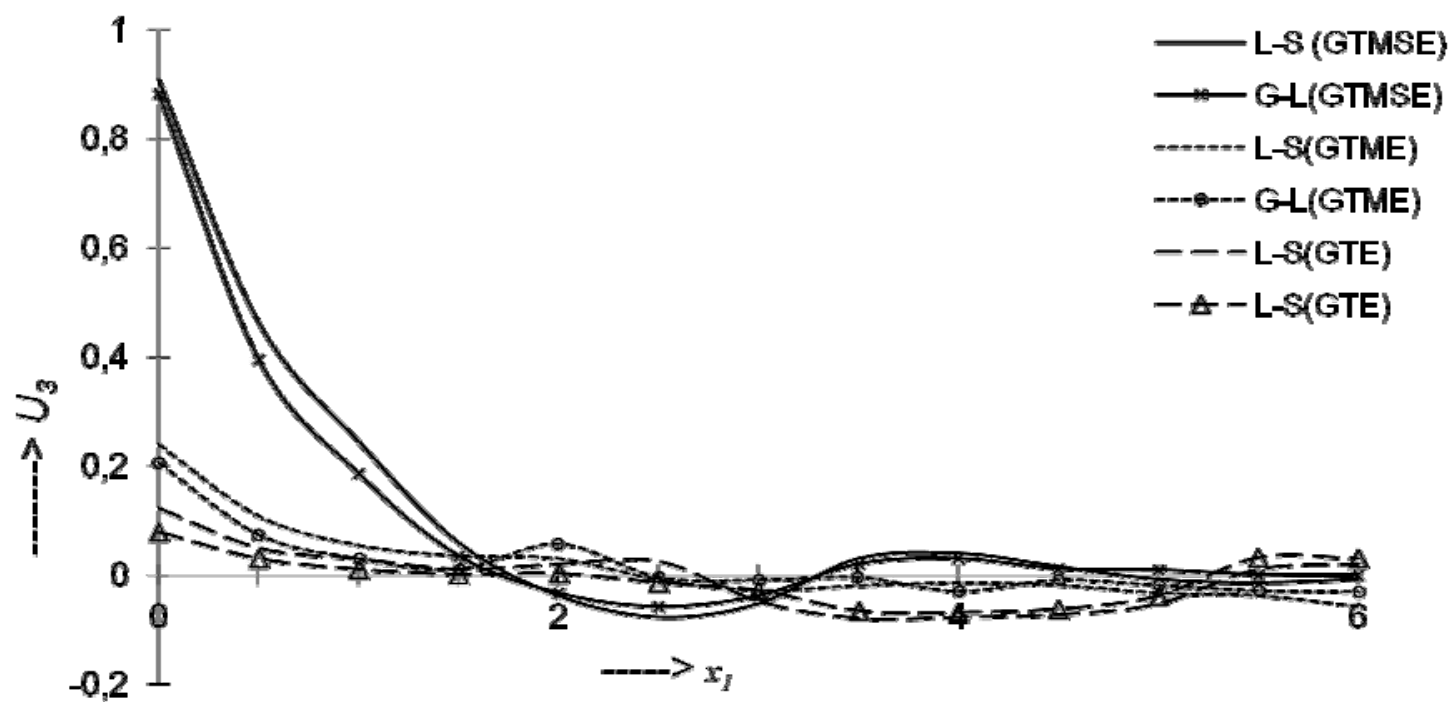

Fig.2. Variation of normal displacement $U_{3}$.

The behaviour of displacement for both theories (L-S and G-L) in all three media (GTMSE, GTME, GTE) is similar, whereas due to the stretch effect, the value of normal displacement in the GTMSE medium is slightly different as compared to those in the GTME medium for L-S and G-L theories as shown in Fig.2. 
The variations of normal displacement $U_{3}$ for L-S and G-L theories are less in the GTMSE medium as compared to the GTME medium in the range $0<x_{1}<2.5$ and vice-versa in the range and for G-L theory values ar greater in the GTME medium as compared to the GTME medium in the range $0<x_{l}<1.3,4.3<x_{l}<5.9$ and less in the range $1.3<x_{1}<4.3$. The rotational effect on the values of normal displacement for all the three theories has been depicted in Fig.2.

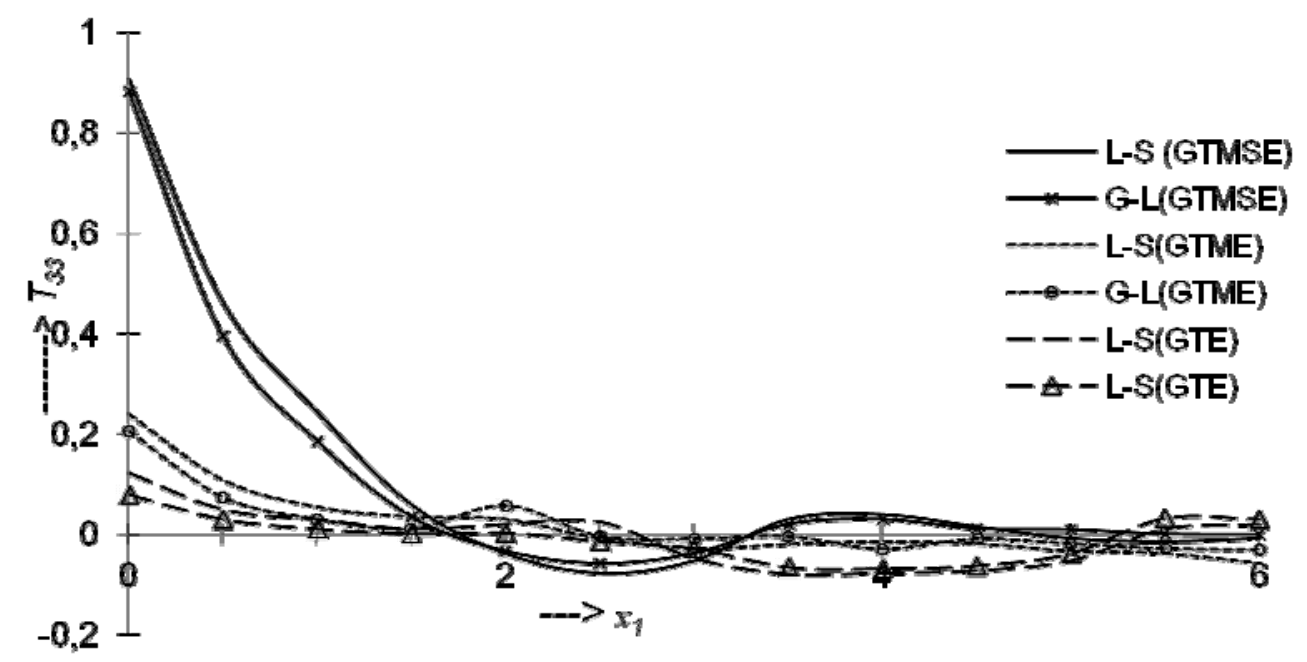

Fig.3. Variation of normal stress $T_{33}$.

The values of normal force stress $T_{33}$ for L-S theory are greater in the range $0<x_{1}<1.3,3<x_{1}<6$ but less in the range $1.3<x_{1}<3.3$ for G-L theory values are less in the range $0<x_{1}<1.25,3<x_{1}<6$ and greater in range $1.25<x_{I}<3.3$ in the TE medium as compared to the MTE medium. Figure 3 depicts these variations after dividing the original values of G-L theory in the GTE medium by 10 to depict the comparison.

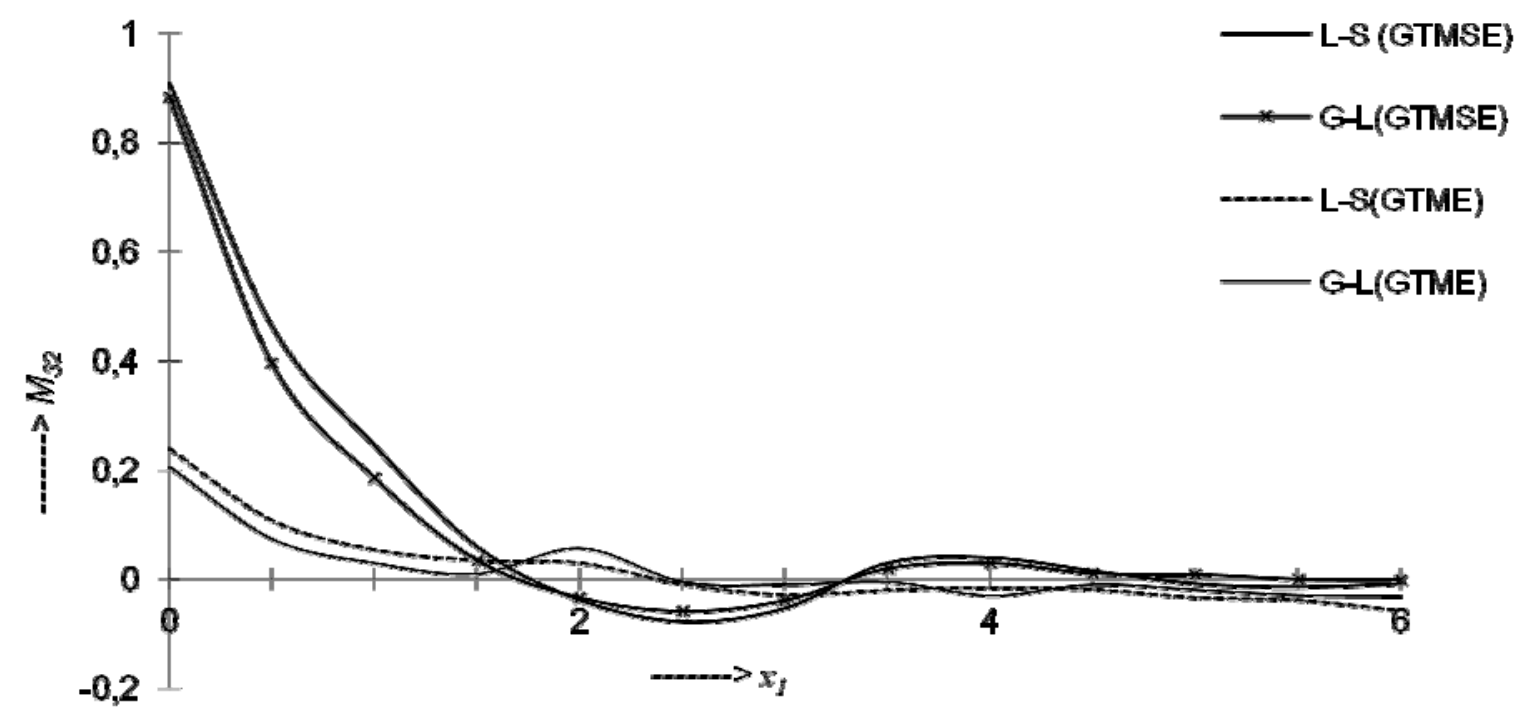

Fig.4. Variations of tangential couple stree $M_{32}$.

Figure 4 shows these variations of normal force stress $T_{33}$ for three different theories in both the media after multiplying the original values of G-L theory in the GMTE medium by $10^{2}$. 


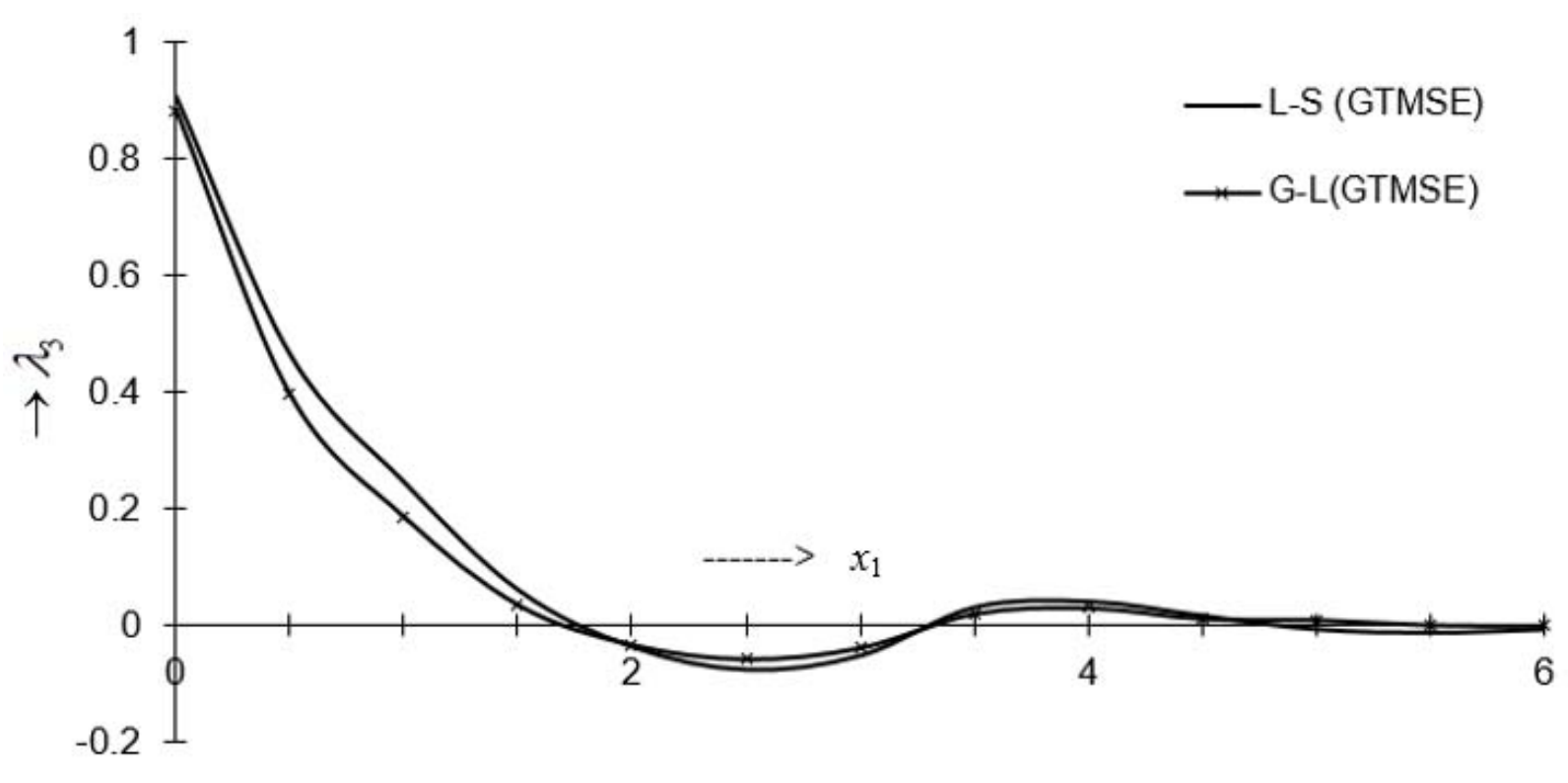

Fig.5. Variations of microstress $\lambda_{3}^{*}$.

The variations of tangential couple stress $\lambda_{3}^{*}$ for G-L theory are different and take place in a greater range as compared to L-S and G-L theories. Also the values for G-L theory are greater as compared to L-S theory in the range $0<x_{1}<0.8$ and $5.3<x_{1}<6$ and are less in the range $0.8<x_{1}<5.3$ as depicted in Fig.5.

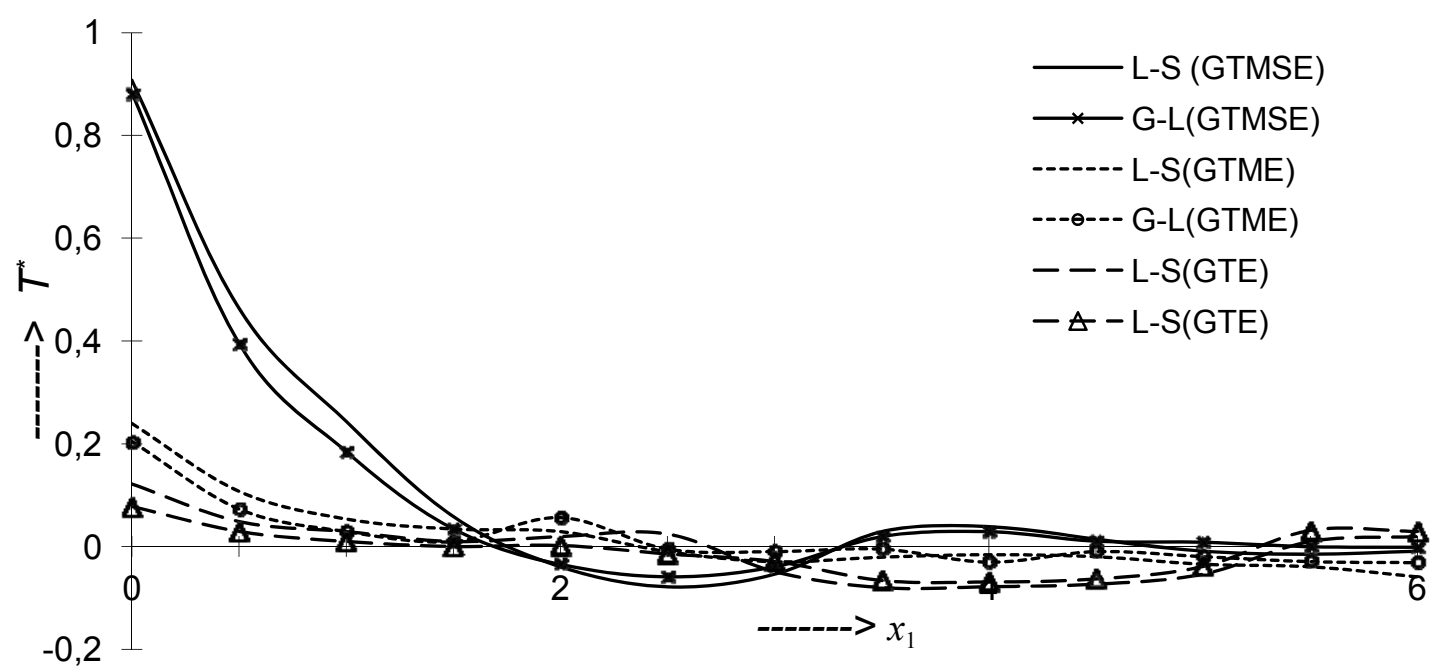

Fig.6. Variation of temperature field $T^{*}$.

The temperature field $\mathrm{T}$ for three different theories in the MTE medium has variations similar to the variations in the TE medium, where the range of variations for L-S theory is greater as compared to G-L theory as depicted in Fig.6. For L-S theory, values of temperature distribution are less in the range $0<x_{1}<1.7$ and $3.5<x_{1}<4.7$ and greater in the range $1.7<x_{1}<3.5$ and $4.7<x_{1}<6$; for G-L theory the values of temperature distribution are less in the range $0<x_{1}<1.5$ and $3.6<x_{1}<4.5$ and greater in the range $1.5<x_{1}<3.6$ and $4.5<x_{1}<6$ in the GTE medium as compared to the GMTE medium. 


\section{Conclusion}

For both L-S and G-L theories, impact of microstretch effect on stress components is more as compared to micropolarity. Additionally it is observed that effect of microstretch on displacement, normal stress, tangential couple stress, microstress and temperature distribution depends upon the distance. Furthermore variation in all these components shows oscillatory behavior as we move away from point of application of impulsive force. Using these results, it is possible to investigate the disturbance caused by more general source for practical applications

\section{Nomenclature}

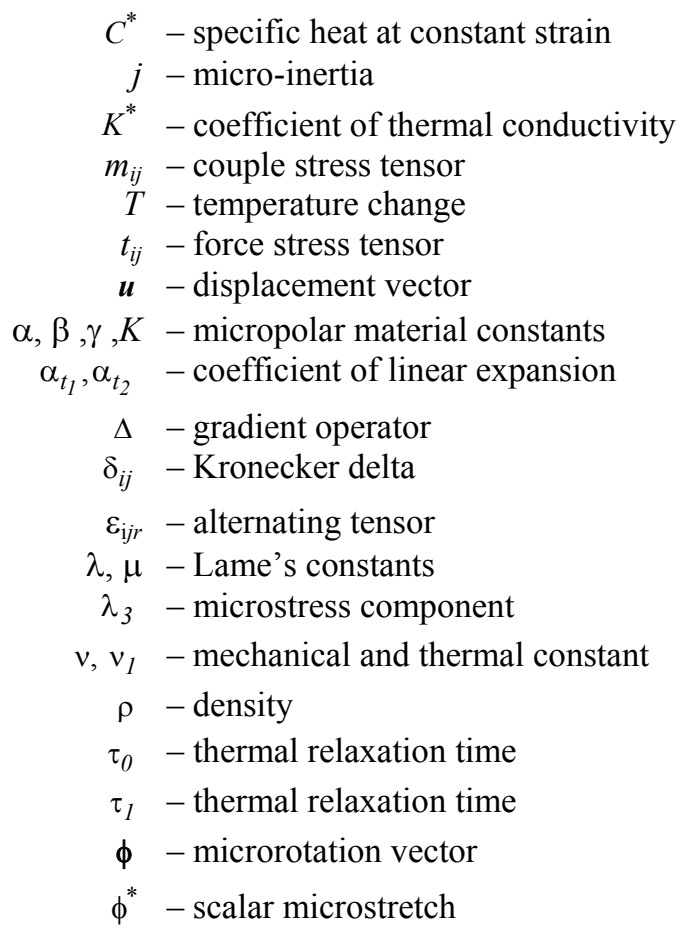

\section{References}

Abbas I.A. and Othman M.I.A. (2012): Plane waves in generalized thermo-microstretch elastic solid with thermal relaxation using finite element method. - Int. J. Thermophys., vol.33, No.12, pp.2407-2423.

Ciarletta M. (1999): A theory of micropolar thermoelasticity without energy dissipation. - J. Thermal Stresses, vol.22, pp.581-594.

Eringen A.C. (1966): Linear theory of micropolar elasticity. - J. Math. Mech., vol.15, pp.909-923.

Eringen A.C. (1970): Foundation of micropolar thermoelasticity. - Course of Lectures No.23.- Verlag: Springer.

Eringen A.C. (1971): Micropolar elastic solids with stretch. - Ari. Kitabevi Matbassi, vol.24, pp.1-18.

Eringen A.C. (1984): Plane waves in non-local micropolar elasticity. - Int. J. Eng. Sci., vol.22, pp.1113-1121.

Eringen A.C. (1990): Theory of thermo-microstretch elastic solids. - Int. J. Eng. Sci., vol.28, pp.1291-1301.

Green A.E. and Lindsay K.A. (1972): Thermoelasticity. - J. Elasticity, vol.2, pp.1-7.

Green A.E. and Naghdi P.M. (1993): Thermoelasticity without energy dissipation. - J. Elasticity, vol.31, pp.189-208. 
Kumar R. and Partap G.(2009): Axisymmetric free vibrations in microstretch thermoelastic homogenous isotropic plate. - Int. J. of App. Mech. and Eng., vol.14, No.1, pp.211-237.

Kumar R. and Deswal S. (2001): Disturbance due to mechanical and thermal sources in a generalized thermomicrostretch elastic space. - Sadhana, vol.26, No.6, pp.529-547.

Kumar R., Singh R. and Chadha T.K. (2003): Plane strain problem in microstretch elastic solid. - Sadhana, vol.28, No.6, pp.975-990.

Lord H.W. and Shulman Y. (1967): A generalized dynamical theory of thermoelasticity. - J. Mech. Phys. Solid, vol.15, pp.299-309.

Lotfy K. and Othman M.I.A. (2012): Effect of rotation on plane waves in generalized thermo-microstretch elastic solid with a relaxation time. - Meccanica, vol.47, pp.1467-1486.

Nowacki W. (1966): Couple stresses in the theory of thermoelasticity. - Proc. ITUAM Symposia, Vienna, SpringerVerlag, pp.259-278.

Othman M.I.A. and Lotfy K. (2010): On the plane waves of generalized thermo-microstretch elastic half-space under three theories. - Int. Communications in Heat and Mass Transfer, vol.37, No.2, pp.192-200.

Othman M.I.A. Atwa S.Y., Jahangir A. and Khan A. (2013): Effect of magnetic field and rotation on generalized thermo microstretch elstic solid with mode-I crack under Green Naghdi theory. - Computational Mathematics and Modeling, vol.24, No.4, pp.556-591.

Press W.H. et al. (1986): Numerical Recipes in FORTRAN 2nd Edition. - Cambridge: Cambridge University Press.

Received: April 27, 2014

Revised: May 22. 2015 